\title{
Preface to the special issue on ultrafine-grained materials
}

\author{
Suveen N. Mathaudhu $\cdot$ Xiaoxu Huang $\cdot$ Hyoung Seop Kim • \\ Terence G. Langdon • Terry C. Lowe • Ruslan Z. Valiev • \\ Xiaolei Wu $\cdot$ Michael Zehetbauer • Yuntian T. Zhu
}

Received: 10 July 2012/ Accepted: 10 July 2012/Published online: 24 July 2012

(C) Springer Science+Business Media, LLC 2012

This issue marks the fourth special issue on ultrafinegrained (UFG) materials to be published in the Journal of Materials Science. The scientific works given here represent selected papers from the Seventh International Symposium on Ultrafine-Grained Materials (UFG VII), which was held in Orlando, FL, USA from March 11-15, 2012. The symposium attracted 160 abstracts from 25 countries, making it the largest symposium at the 2012 TMS Annual Meeting and Exhibition. The UFG symposium provides a forum on the topics of fabrication of UFG and nanocrystalline materials including conventional and emerging technologies and advancements, fundamental issues in severe plastic deformation (SPD) processing and SPD-

\section{S. N. Mathaudhu}

Materials Science Division, U.S. Army Research Office,

Durham, NC, USA

e-mail: suveen.n.mathaudhu.civ@mail.mil

\section{Huang}

Risø National Lab, Roskilde, Denmark

e-mail: xihu@dtu.dk

\section{H. S. Kim}

Department of Materials Science and Engineering, POSTECH

(Pohang University of Science and Technology), Hyoja-dong,

Pohang, Gyungbuk 790-784, Korea

e-mail: hskim@postech.edu

URL: http://hskim.postech.ac.kr

\section{T. G. Langdon}

Department of Aerospace \& Mechanical Engineering, University

of Southern California, Los Angeles, CA 90089-1453, USA

e-mail: langdon@usc.edu

T. C. Lowe

Metallicum Inc., a Manhattan Scientifics Inc. Company,

Santa Fe, NM, USA

e-mail: terry@mhtx.com processed materials, UFG and nanocrystalline microstructure evolution, mechanical and physical properties, deformation mechanisms, superplasticity, joining and bonding, computational and analytical modeling, structural and functional applications, and thermal stability.

Ultrafine-grained materials include all classes of metals and composites with domains less than $1 \mu \mathrm{m}(1,000 \mathrm{~nm})$ in size. Nanocrystalline materials, typically defined to have domain sizes less than $100 \mathrm{~nm}$, are certainly a subcategory within UFG materials. Within the community represented at the bi-yearly UFG meeting, there is an added expectation that the materials will be "bulk" in nature such that physical and mechanical properties can be measured.

\section{R. Z. Valiev}

Institute of Physics of Advanced Materials, Ufa State Aviation Technical University, 12 K. Marx Street, Ufa 450000, Russia e-mail: rzvaliev@mail.rb.ru

\section{$\mathrm{X} . \mathrm{Wu}$}

State Key Laboratory of Nonlinear Mechanics \& Materials Research Center, Institute of Mechanics, Chinese Academy of Sciences, 15 BeiSiHuan West Road, Beijing 100190, China e-mail:xlwu@imech.ac.cn

M. Zehetbauer

Faculty of Physics, University of Vienna, Boltzmanngasse 5, 1090 Vienna, Austria

e-mail: michael.zehetbauer@univie.ac.at

Y. T. Zhu $(\bowtie)$

Department of Materials Science \& Engineering, North Carolina State University, Raleigh, NC 27695-7919, USA

e-mail: ytzhu@ncsu.edu 
The largest contributions by number have been from researchers investigating SPD of metals and alloys via methods, such as equal-channel angular extrusion/pressing (ECAE/ECAP), high-pressure torsion (HPT), accumulative roll bonding (ARB), and a number of other similar methods that can rapidly refine grain structures to submicrocrystalline regimes with the additional benefit of no change in workpiece cross-section, as would occur during conventional wrought processing, such as rolling, drawing, extrusion or forging. Proportional to the high number of researchers in this area, the bulk of the papers given here are related to SPD processing methods. Another general approach to synthesizing UFG materials is via powder processing routes. Typical approaches here include mechanical alloying and cryomilling, which severely deform powder materials, followed by consolidation methods that retain the nanocrystalline microstructures obtained. Recent consolidation methods utilized include hot-isostatic pressing (HIP) and spark plasma sintering (SPS), however, there has been a recent trend towards synthesis of powders with thermal stability that can be consolidated through conventional methods at elevated temperatures with little grain growth. Such advancements should open up the door to powder metallurgy alloys with tailored microstructures and properties.

The UFG community continues to grow rapidly, and papers represented here highlight the timely development of new processing methods, computational capacity for simulation tools, and concurrent development of atomicscale resolution microscopy tools, 3D-microscopy and in situ characterization. These methods have led to unprecedented scientific advancements that are expected to extensively improve material properties and thus facilitate more applications in the near future. 